\title{
ORIGINAL ARTICLE \\ Diurnal blood pressure and urine production in acute spinal cord injury compared with controls
}

\author{
MY Goh ${ }^{1,2,3}$, MS Millard ${ }^{1,4}$, ECK Wong ${ }^{1}$, DJ Brown ${ }^{3}$, AG Frauman ${ }^{1,2}$ and CJ O’Callaghan ${ }^{1,2}$
}

Study design: This is a prospective observational study.

Objectives: The objective of this study was to determine time-dependent changes in diurnal blood pressure (BP) and urine production in acute spinal cord injury (SCI).

Setting: This study was conducted in a specialist, state-based spinal cord service in Victoria, Australia.

Methods: Consenting patients admitted consecutively with acute SCI were compared with patients confined to bed rest while awaiting surgery and with mobilising able-bodied controls. Participants underwent ambulatory BP monitoring (ABPM), measurement of diurnal urine production and rated orthostatic symptoms over 1 year. Participants with night:day systolic BP (SBP) $<90 \%$ were classified as dippers, $90-100 \%$ as non-dippers and $>100 \%$ as reverse dippers.

Results: Participants comprised tetraplegics ( $n=47,40.0 \pm 17.3$ years), paraplegics $(n=35,34.4 \pm 13.9$ years), immobilised ( $n=18,30.9 \pm 11.3$ years) and mobilising $(n=44,33.1 \pm 13.5$ years) controls. At baseline, 24-h BP was significantly lower in tetraplegics $(111.8 \pm 1.9 / 62.1 \pm 1.1 \mathrm{~mm} \mathrm{Hg})$ but not in paraplegics $(116.7 \pm 1.4 / 66.0 \pm 1.1 \mathrm{~mm} \mathrm{Hg})$, compared with controls $(117.1 \pm 1.3 / 69.1 \pm 1.1 \mathrm{~mm} \mathrm{Hg})$, adjusting for gender. This difference was not observed at 1 year. The average night:day SBP in mobilising controls was $86.1 \pm 0.7 \%$, differing from paraplegics $(94.0 \pm 1.5 \%, P<0.001)$ and tetraplegics $(101.5 \pm 1.5 \%$, $P<0.001)$. Urine production in tetraplegics and paraplegics did not fall at night compared with the day. Abnormal diurnal BP and orthostatic symptoms in tetraplegics persisted throughout the study. Nocturnal hypertension was observed in $27 \%(n=9)$ of tetraplegics, of whom only 2 had day hypertension. All mobilising controls with nocturnal hypertension $(n=6,14 \%)$ had day hypertension.

Conclusion: People with $\mathrm{SCl}$ have a high prevalence of isolated nocturnal hypertension, reverse dipping, orthostatic intolerance and nocturnal polyuria. Cardiovascular risk management and assessment of orthostatic symptoms should include ABPM.

Spinal Cord (2017) 55, 39-46 doi:10.1038/sc.2016.100; published online 28 June 2016

\section{INTRODUCTION}

Disruption of the neural pathways that regulate cardiovascular function markedly impairs blood pressure (BP) control. The clinical consequences of autonomic damage in spinal cord injury (SCI) are well recognised and include severe hypotension and persistent bradycardia, large daily fluctuations in BP, autonomic dysreflexia and potentially premature arterial disease and increased cardiovascular risk. ${ }^{1-4}$

When autonomic disease affects the able-bodied population, it produces similarly severe clinical impairment. In this cohort, measurement of BP during ambulation has been proven to be invaluable in the management of symptoms, control of cardiovascular risk and identification of potential mechanisms, which may contribute to orthostatic intolerance. In particular, ambulatory BP monitoring (ABPM) has been instrumental in identifying the disruptions of diurnal variation of $\mathrm{BP}$ and the role that elevated nocturnal pressures may have in nocturnal diuresis and natriuresis, which have been proposed to contribute to postural hypotension. ${ }^{5-7}$

The role of ambulatory monitoring in SCI is less well defined. Most investigations have been restricted to relatively healthy volunteers and usually excluded participants with cardiovascular disease and significant BP symptoms. ${ }^{8-11}$ In general, these studies found that SCI was characterised by loss of the usual diurnal variation in BP. Only recently have investigations included symptomatic patients, ${ }^{12,13}$ including our study, which found that patients with orthostatic symptoms had similar or even more extreme abnormalities than asymptomatic patients-particularly in the period soon after injury. ${ }^{14}$

It is unknown whether abnormalities of diurnal BP and urine production, which are observed soon after SCI, persist over time or whether they contribute to symptoms of orthostatic hypotension. In this study, we examined the 24-h ambulatory BP patterns of patients admitted consecutively to our SCI service. We compared results in SCI with control participants who were able-bodied and independently mobilising and with a group of similarly able-bodied participants who were immobilised while awaiting surgical procedures and followed them up over a year.

\section{METHODS}

Screening was performed on patients who were consecutively admitted to the SCI unit between February 2010 and December 2013 for acute, traumatic SCI, and eligible consenting participants were enrolled. Immobilised control participants were otherwise healthy patients confined to bed rest while awaiting orthopaedic surgery. Healthy, mobilising able-bodied control participants

${ }^{1}$ Department of Clinical Pharmacology, Austin Hospital, Heidelberg, Victoria, Australia; ${ }^{2}$ Department of Medicine, University of Melbourne, Parkville, Victoria, Australia; ${ }^{3}$ The Spinal Research Institute, Heidelberg, Victoria, Australia and ${ }^{4}$ Victorian Spinal Cord Service, Heidelberg, Victoria, Australia

Correspondence: Dr MY Goh, Department of Clinical Pharmacology, Austin Hospital, Level 5 Lance Townsend Building, PO Box 5555, Heidelberg, Victoria 3084, Australia. E-mail: Min.Goh@austin.org.au

Received 16 November 2015; revised 22 May 2016; accepted 28 May 2016; published online 28 June 2016 
without known cardiovascular conditions were recruited by advertisement. Comparisons were made between tetraplegic, paraplegic (including high and low paraplegics) and immobilised and mobilising able-bodied participants at each time point and over time within each group.

Participants were interviewed and medical records were reviewed to characterise demographic details (age, gender) and details of SCI (date, mechanism of injury, level and completeness of injury), medical conditions and concurrent medications. Level and completeness of injury were extracted from the medical history, as assessed by physical examination and recorded according to the International Standards for Neurological Classification of Spinal Cord Injury during the admission. ${ }^{15}$ Imaging was not used for classification. Participants were designated as tetraplegics if their lesion was in the cervical region, and as paraplegics if their lesion was in the thoracic, lumbar or sacral region; paraplegics were further classified as high paraplegics if their lesion was between $\mathrm{T} 1$ and $\mathrm{T} 6$ or as low paraplegics if their lesion was below $\mathrm{T} 6 \mathrm{r}^{1,11,16,17}$

Measurements included ABPM, quantification of diurnal urine output by completion of a diary and quantification of orthostatic symptoms on a rating scale. Study assessments were undertaken for SCI groups after initial mobilisation. Participants also had another assessment performed 1 year after mobilisation, and tetraplegic participants had additional assessments at 2, 4 and 6 months after mobilisation. Assessments were delayed if an active acute medical condition precluded performance of investigations. Control groups underwent assessments at baseline and 1 year. During the course of the study, some participants underwent sleep studies either as part of clinical assessment or through participation in a concurrent study examining sleep apnoea in SCI.

\section{Ambulatory BP monitoring}

Ambulatory BPs were obtained using the $\operatorname{Card}(\mathrm{X})$ plore monitor (Meditech, Budapest, Hungary), with an appropriately sized cuff, worn by each participant for about $24 \mathrm{~h}$. Measurements were taken half-hourly during the day (06002200 hours) and hourly at night (2200-0600 hours). Results were analysed according to mean 24-h, day (1000-2000 hours) and night (0000-0600 hours) values for systolic BP (SBP), diastolic BP (DBP) and pulse rate. Day and night were defined by fixed clock time intervals to eliminate transition periods in the morning and evening when there is considerable variation in $\mathrm{BP}^{18}$ and also in postural change among groups. This method has been found to correspond well with actual awake and asleep periods. ${ }^{19}$ This is especially applicable to the SCI population who may dress in bed after getting upright for the morning routine and may return to bed for a duration before going to sleep for the night; hence, there is a period of time over which postural change occurs.

The extent of nocturnal dip in BP was calculated as the ratio of night-to-day SBP, expressed as a percentage. Participants with a night:day SBP of $<90 \%$ (the normal pattern) were classified as 'dippers', $90-100 \%$ as 'non-dippers' and $>100 \%$ as 'reverse dippers'. ${ }^{20}$ Hypertension was defined as a $24-\mathrm{h}$ $\mathrm{BP}>130 / 80 \mathrm{~mm} \mathrm{Hg}$, day hypertension as $\mathrm{BP}>135 / 85 \mathrm{~mm} \mathrm{Hg}$ and nocturnal hypertension as $\mathrm{BP}>120 / 70 \mathrm{~mm} \mathrm{Hg}$, according to clinical guidelines. ${ }^{21}$

\section{Postural BP measurement}

Participants or their carers were requested to obtain BP measurements on the ambulatory BP monitor, by pressing a button. They were instructed to obtain two supine and three upright BPs, a minute apart, on first arising from bed the morning after the monitor was applied. Participants were considered upright when either standing up or sitting up in a chair for those with SCI who were unable to stand. Orthostatic hypotension was defined as a fall in BP of 20/10 $\mathrm{mm} \mathrm{Hg}$ within $3 \mathrm{~min}$ of assuming the upright position. ${ }^{22}$

\section{Urine measurements}

Urine output was recorded over 3 consecutive days, generally within a week of BP monitoring. Participants were instructed to record the time that they void or empty their catheter bag on waking on the first day (Day 1) and then to record urine volumes and times at each void or each time the catheter bag was emptied, until and including the measurement on first waking on Day 4. A measuring jug was provided for measurement of voided urine or urine emptied from the catheter bag. For the purposes of this study, the terms urine output and urine production are used interchangeably and refer to the volume of urine measured from voiding or emptying of the catheter bag. The day and night rates of urine output were calculated by dividing the volume of urine by the time between measurements and expressed as $\mathrm{ml}$ per minute. Measurements reported were average values for the 3 days and nights. For each group, comparisons were made between the rate of urine output during the day and the night to determine whether the rate of urine output at night was significantly different from the rate during the day.

\section{Symptom assessment}

Participants were asked to rate their symptoms of orthostatic intolerance for the past week on a scale of 0 for none at all to 10 for the worst possible. Symptoms of orthostatic intolerance were explained as symptoms (lightheadedness, dizziness) pertaining to low BP often arising from postural change or remaining upright for some time and relieved with tilting backwards, lifting up legs or lying down.

\section{Statistical analysis}

Analysis was performed using the Microsoft Excel 2007 (Microsoft, Redmond, WA, USA) and R software (http://www.r-project.org). For continuous variables such as BP and pulse rate, linear mixed models were used to examine the interaction between group and time point, adjusting for gender, with subjects as random effects. The interaction between group and time point was included in the model, as we aimed to examine the differences between groups at baseline and at 1 year, as well as the change over time for each group, with the hypothesis that the controls would not differ over time while the other groups may. We report the overall test of the interaction from this model, and pairwise comparisons for group at each time point were made using the Tukey's Honestly Significant Difference test. Paired $t$-tests were used to compare the day and night continuous variables (BP, pulse rate and urine output rates) within each group. The chi-square test was used for categorical variables (such as gender and dipping status). The Kruskal-Wallis and Wilcoxon signed-rank tests and Spearman's correlation test were used to compare non-parametric variables. Significance was set at $P<0.05$. Range intervals represent s.e.m.

\section{Statement of ethics}

We certify that all applicable institutional and governmental regulations concerning the ethical use of human volunteers were followed during the course of this research. The protocol for this study was approved by the Human Research Ethics Committee of Austin Health.

\section{RESULTS}

\section{Study group characteristics}

Of the 116 tetraplegic and 73 paraplegic patients with traumatic SCI who were screened, 49 and 23, respectively, were ineligible for reasons including age, medical instability or death. Participation was declined by a further 19 and 15 tetraplegic and paraplegic patients, respectively, resulting in recruitment of 48 tetraplegics and 35 paraplegics $(72 \%$ and $70 \%$ of eligible participants, respectively). One tetraplegic participant was later withdrawn because of lack of data, leaving 47 tetraplegics. Of these 47 tetraplegics, 12 had not enrolled at the initial visit, predominantly because other clinical problems precluded participation or they were initially reluctant to engage in the study. Analyses performed including and excluding participants who did not enrol in the first visit were not different. There were significantly fewer male participants in the mobilising control group (Table 1).

Patients who declined to participate in the study did not differ significantly from the study group in age, gender or neurological category of SCI.

\section{Medications}

At baseline, one tetraplegic patient was taking an ACE-inhibitor (angiotensin-converting enzyme inhibitor) and four paraplegic patients were taking antihypertensive agents (beta blockers $(n=2)$, 
Table 1 Characteristics of the study group

\begin{tabular}{|c|c|c|c|c|}
\hline & $\begin{array}{c}\text { Tetraplegics } \\
\mathrm{n}=47\end{array}$ & $\begin{array}{l}\text { Paraplegics }^{\mathrm{a}} \\
\mathrm{n}=35\end{array}$ & $\begin{array}{l}\text { Immobilised } \\
\mathrm{n}=18\end{array}$ & $\begin{array}{c}\text { Mobilising controls } \\
\qquad \mathrm{n}=44\end{array}$ \\
\hline Age, years & $40.0 \pm 17.3$ & $35.4 \pm 13.9$ & $30.9 \pm 11.3$ & $33.1 \pm 13.5$ \\
\hline Range & $16-70$ & $16-63$ & $19-50$ & $16-65$ \\
\hline Male & 42 (89\%) & $28(80 \%)$ & $14(78 \%)$ & $25(57 \%)$ \\
\hline \multirow[t]{3}{*}{ Neurological classification } & C1-4, ABC: 20 & T1-6, ABC: 13 & & \\
\hline & C5-8, ABC: 20 & T7-S2, ABC: 20 & & \\
\hline & D: 7 & D: 2 & & \\
\hline $\mathrm{SCl}$ to mobilisation (days) & $16.6 \pm 12.3$ & $13.5 \pm 10.1$ & & \\
\hline Median & 13 & 12 & & \\
\hline Range & $2-27$ & $1-25$ & & \\
\hline $\mathrm{SCl}$ to ABPM (days) & $28.3 \pm 15.0$ & $27.1 \pm 13.4$ & & \\
\hline Median & 26 & 24.5 & & \\
\hline Range & $6-92$ & $6-58$ & & \\
\hline \multicolumn{5}{|l|}{ Co-morbidities } \\
\hline Smoking (current) & $9(19 \%)$ & $6(17 \%)$ & $4(22 \%)$ & $1(2 \%)$ \\
\hline Hypertension at baseline & 6 & 3 (+1 suspected $\mathrm{WCH}$ ) & 0 & 0 \\
\hline Diabetes mellitus & 3 (type 2) & 1 (type 1) & 0 & 0 \\
\hline Dyslipidaemia & 2 & 0 & 0 & 2 \\
\hline
\end{tabular}

Abbreviations: ABPM, ambulatory blood pressure monitoring; ABC, American Spinal Injury Association Impairment Scale (AIS) A, B or C; AF, atrial fibrillation; D, AIS D; MVP, mitral valve prolapse; OSA, obstructive sleep apnoea; SCl, spinal cord injury; SVT, supraventricular tachycardia; WCH, white coat hypertension.

Age and time periods shown as mean \pm s.d.

aHigh and low paraplegics.

ACE-inhibitors $(n=1)$ and calcium channel blockers $(n=1)$ before admission. These patients all continued antihypertensive therapy for the duration of the study.

Antihypertensive treatments at the 1-year assessment included an ACE-inhibitor for one mobilising control and one tetraplegic patient and a calcium channel blocker and ACE-inhibitor for one paraplegic patient. Other cardiovascular medications included clonidine taken as an analgesic by one paraplegic patient. One tetraplegic patient was taking fludrocortisone.

\section{Immobilised controls}

Immobilised controls were admitted under the orthopaedic unit for lower limb fractures $(n=10)$, vertebral fractures without spinal cord involvement $(n=6)$ or lower limb soft tissue injury $(n=2)$. They were confined to bed rest for 1-11 days (mean 2.6 days, median 2 days) before ABPM.

\section{Population retention}

Loss to follow-up over time resulted in about half the participants attending the final assessment in each group. Of participants who were lost to follow-up, $29-56 \%$ in each group were not contactable, about $33 \%$ in each group declined assessment and about $20 \%$
(0 for immobilised controls) were in a geographically different location, making follow-up not feasible. Baseline BP, urine output rates and symptom scores were not significantly different between those who did and did not attend the final assessment.

\section{BP: baseline}

Tests of interaction for each parameter are reported in Table 2. Where the interaction is small, we expect the estimated differences between groups at each time point to be similar. Where the interaction is larger, we expect the estimated differences to be more variable. The pairwise comparisons at each time point help characterise these differences, in reference to our hypothesis.

Consistent with their age and paucity of other co-morbidities, average BPs across all groups were substantially lower than diagnostic levels for hypertension ${ }^{21}$ (Table 3). Nevertheless, among the mobilising control group, average day and night BPs exceeded thresholds for hypertension in $19 \%$ and $14 \%$ of participants, respectively. In the control group, average night:day SBP was $86.1 \%$ and none were reverse dippers. In control participants, $24-\mathrm{h}$ SBP was significantly higher in male than in female participants, but no other BP or pulse rate parameter was affected by gender 
$(119.3 \pm 1.7 / 70.2 \pm 1.5 \mathrm{~mm} \mathrm{Hg} \quad$ vs $\quad 113.9 \pm 2.0 / 67.6 \pm 1.6 \mathrm{~mm} \mathrm{Hg}$, $P=0.044$ and $P=0.256$ for SBP and DBP, respectively).

Compared with the controls, immobilised participants had significantly higher average BPs (Table 3), with average day and night BPs exceeding the limit for hypertension in $33 \%$ and $53 \%$ of participants. The night:day SBP did not reach statistical significance when compared with that of control participants $(P=0.087)$.

Table 2 Tests of interaction from linear mixed models

\begin{tabular}{lll}
\hline & $F_{3,51}$ & P-value \\
\hline Systolic BP & & \\
$24 \mathrm{~h}$ & 0.533 & 0.662 \\
Day & 0.733 & 0.537 \\
Night & 0.673 & 0.572 \\
Night:day (\%) & 1.530 & 0.218 \\
& & \\
Diastolic BP & & \\
$24 \mathrm{~h}$ & 1.524 & 0.220 \\
Day & 1.787 & 0.161 \\
Night & 2.231 & 0.096 \\
& & \\
Pulse rate & & 0.017 \\
24 h & 3.730 & 0.076 \\
Day & 2.433 & 0.130 \\
$\quad$ Night & 1.973 & \\
\hline Abbreviation: BP, blood pressure. & &
\end{tabular}

Abbreviation: $\mathrm{BP}$, blood pressure.
BPs in paraplegic participants did not differ from controls, but they were significantly lower than that of the immobilised participants (Table 3). Average day and night pressures were higher than limits for hypertension in $8 \%$ and $19 \%$ of participants. The night:day SBP $(94 \%)$ was significantly different from that of the controls $(P<0.001)$, and $6(23 \%)$ participants were reverse dippers.

Tetraplegics had significantly lower day BP than all other groups, whereas night pressures were not significantly different from that of mobilising controls or paraplegics (Table 3). Mean 24-h BPs were significantly lower than that of mobilising controls after adjusting for gender but not different from that of paraplegics. Average BPs exceeded limits for hypertension in $8 \%$ of participants during the day but in $27 \%$ of participants at night. The night:day SBP $(101.5 \%)$ was significantly higher than the night:day SBP of each of the other groups. Reverse dipping was found in 18 (55\%) tetraplegics.

\section{Subgroups}

Although the night:day SBP in all paraplegic participants was significantly different from that of tetraplegics, when analysed according to level of paraplegia, high paraplegia $(n=9,98.3 \pm 2.5 \%)$ was not significantly different from tetraplegia $(n=33,101.5 \pm 1.5 \%)$ $(P=0.753)$, whereas low paraplegia $(n=17,91.8 \pm 1.7 \%)$ differed significantly from tetraplegia $(P<0.001)$. Both high and low paraplegia were significantly different from controls $(n=43,86.1 \pm 0.7 \%)$ $(P<0.001$ and $P=0.033$ for high and low paraplegia, respectively, compared with controls). Differences in the day and night systolic and diastolic BPs for each subgroup are shown in Figure 1.

The night:day SBP for complete tetraplegics $(n=11,103.3 \pm 3.0 \%)$ was not significantly different from that of incomplete tetraplegics

Table 3 Diurnal blood pressure and pulse rate at baseline

\begin{tabular}{|c|c|c|c|c|}
\hline & $\begin{array}{c}\text { Tetraplegics } \\
\mathrm{n}=33\end{array}$ & $\begin{array}{c}\text { Paraplegics }^{\mathrm{a}} \\
\mathrm{n}=26\end{array}$ & $\begin{array}{c}\text { Immobilised } \\
\quad \mathrm{n}=15\end{array}$ & $\begin{array}{c}\text { Mobilising controls } \\
\qquad n=43\end{array}$ \\
\hline \multicolumn{5}{|c|}{ Systolic BP (mm Hg) } \\
\hline $24 \mathrm{~h}$ & $111.8 \pm 1.9 *$ & $116.7 \pm 1.4^{\#}$ & $126.6 \pm 3.2^{*}$ & $117.1 \pm 1.3$ \\
\hline Day & $110.9 \pm 2.2^{* *, \# \#, \int}$ & $119.6 \pm 1.7^{\#}$ & $131.7 \pm 3.5^{*}$ & $123.0 \pm 1.5$ \\
\hline Night & $112.0 \pm 2.0^{\#}$ & $112.2 \pm 1.6^{\#}$ & $119.5 \pm 3.0^{* *}$ & $105.7 \pm 1.4$ \\
\hline Night:day (\%) & $101.5 \pm 1.5^{* *, \# \#, \iint}$ & $94.0 \pm 1.5^{* *}$ & $91.1 \pm 1.6$ & $86.1 \pm 0.7$ \\
\hline Reverse dippers^ & $18(55 \%)$ & $6(23 \%)$ & $1(7 \%)$ & $0(0 \%)$ \\
\hline \multicolumn{5}{|c|}{ Diastolic BP $(\mathrm{mm} \mathrm{Hg})$} \\
\hline $24 \mathrm{~h}$ & $62.1 \pm 1.1 * *$ & $66.0 \pm 1.1^{\#}$ & $72.8 \pm 1.8$ & $69.1 \pm 1.1$ \\
\hline Day & $63.1 \pm 1.2^{* *, \# \#, \int}$ & $70.0 \pm 1.5^{*}$ & $75.0 \pm 2.2$ & $74.5 \pm 1.2$ \\
\hline Night & $60.9 \pm 1.2^{\#}$ & $61.4 \pm 1.2^{\#}$ & $69.0 \pm 1.8^{* *}$ & $58.3 \pm 1.2$ \\
\hline \multicolumn{5}{|l|}{ Hypertension } \\
\hline $24 \mathrm{~h}$ & $2(6 \%)$ & $0(0 \%)$ & $6(40 \%)$ & $7(16 \%)$ \\
\hline Day & $3(8 \%)$ & $2(8 \%)$ & $5(33 \%)$ & $8(19 \%)$ \\
\hline Night & $9(27 \%)$ & $5(19 \%)$ & $8(53 \%)$ & $6(14 \%)$ \\
\hline \multicolumn{5}{|l|}{ Pulse rate (b.p.m.) } \\
\hline $24 \mathrm{~h}$ & $68.4 \pm 1.9 \iint$ & $83.4 \pm 2.1^{* *, \# \#}$ & $68.9 \pm 2.6$ & $70.5 \pm 1.3$ \\
\hline Day & $71.0 \pm 1.9 \iint$ & 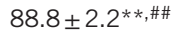 & $72.8 \pm 2.6$ & $75.0 \pm 1.4$ \\
\hline Night & $64.0 \pm 2.1 \int$ & $74.5 \pm 2.3^{* *}, \#$ & $63.1 \pm 3.0$ & $61.7 \pm 1.6$ \\
\hline
\end{tabular}

Abbreviations: BP, blood pressure; b.p.m., beats per minute.

Values are mean \pm s.e.m.

${ }^{*} P<0.05$ vs mobilising controls, ${ }^{* *} P<0.001$ vs mobilising controls.

$\# P<0.05$ vs immobilised controls, $\# P<0.001$ vs immobilised controls.

$\int P<0.05$ vs paraplegics, $\iint P<0.001$ vs paraplegics.

${ }^{\wedge} P<0.001$ between four groups.

aHigh and low paraplegics. 
a

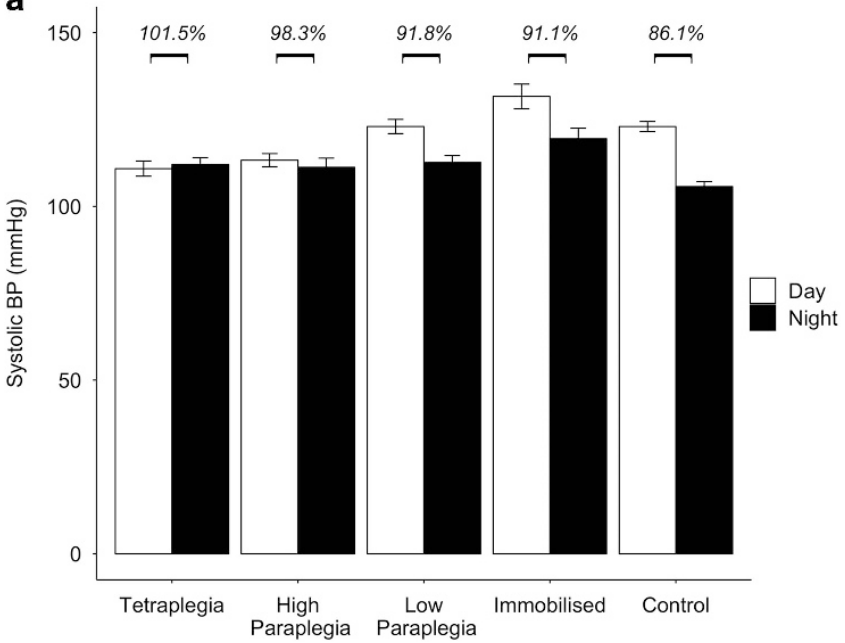

b

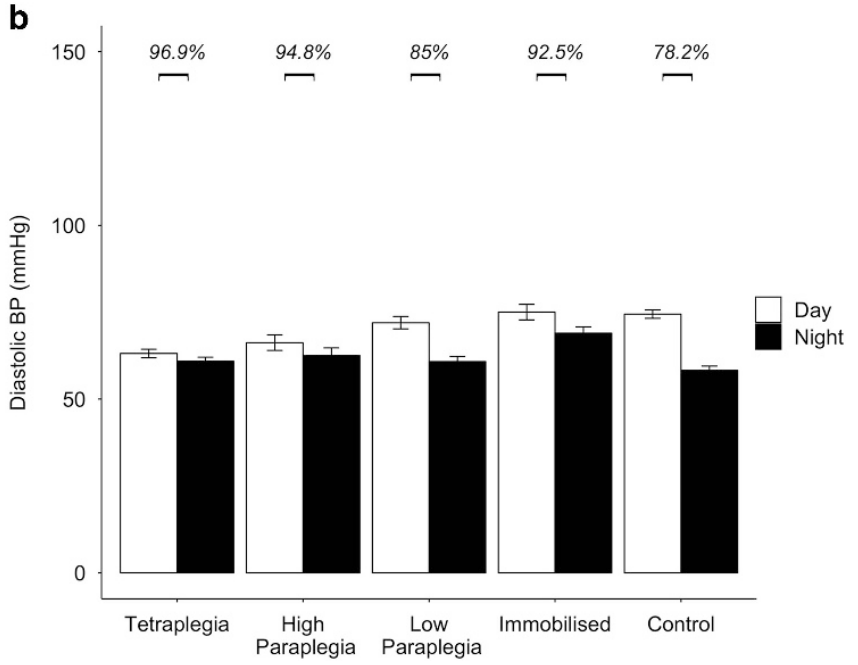

Figure 1 Day and night SBP (a) and DBP (b) for tetraplegics $(n=33)$, high paraplegics $(n=9)$, low paraplegics $(n=17)$, immobilised controls $(n=15)$ and mobilising controls $(n=43)$ at baseline. Error bars represent s.e.m. Percentages above each set of bars represent night:day SBP and night:day DBP in respective graphs.

Table 4 Postural blood pressure and pulse rate changes

\begin{tabular}{lccr}
\hline & Tetraplegics & Paraplegics & Immobilised \\
\hline Baseline & $n=3$ & $n=12$ & $n=0$ \\
Change in SBP (mm Hg) & $-26.1 \pm 3.56$ & $-8.2 \pm 2.44^{*}$ & $n=31$ \\
Change in DBP (mm Hg) & $-21.1 \pm 3.50$ & $-2.4 \pm 1.60^{*}$ & $-0.8 \pm 1.88$ \\
Change in PR (b.p.m.) & $12.8 \pm 6.17$ & $16.6 \pm 3.78^{*}$ & $8.6 \pm 1.51$ \\
& & & $26.0 \pm 1.88$ \\
1 year & $n=12$ & $n=5$ & $n=23$ \\
Change in SBP (mm Hg) & $-13.4 \pm 4.32^{*}$ & $-9.5 \pm 7.18$ & $-1.9 \pm 1.97$ \\
Change in DBP (mm Hg) & $-0.8 \pm 2.20$ & $-3.3 \pm 4.12$ & $4.3 \pm 3.46$ \\
Change in PR (b.p.m.) & $18.0 \pm 4.19$ & $16.7 \pm 6.83$ & $9.7 \pm 2.46$ \\
\hline
\end{tabular}

Abbreviations: b.p.m., beats per minute; DBP, diastolic blood pressure; PR, pulse rate; SBP, systolic blood pressure.

Values are mean \pm s.e.m.

${ }^{\star} P<0.05$ vs mobilising controls.

aHigh and low paraplegics.

$(n=22,100.6 \pm 1.7 \%)(P=0.904)$. Similarly, night:day SBP did not differ significantly between complete paraplegics $(n=19,95.7 \pm 1.7 \%)$ and incomplete paraplegics $(n=7,89.5 \pm 2.6 \%)(P=0.340)$.

The night:day SBP was $103.5 \%$ in tetraplegics, with a sleep study that was positive for obstructive sleep apnoea $(n=16)$ and $104.9 \%$ in those whose sleep study was negative for obstructive sleep apnoea $(n=5)$.

\section{Postural BPs}

Postural BP was not measured at the baseline visit in immobilised controls, because of their need to maintain bed rest, and in many of the tetraplegic participants where it was precluded by clinical circumstances. Compliance with the instruction to measure postural BP was relatively low in the paraplegics and immobilised controls at 1 year.

Compared with controls, the postural fall in SBP was significantly larger in both paraplegic and tetraplegic participants at the baseline and 1-year visits (Table 4). Criteria for orthostatic hypotension were met by $25 \%$ of paraplegic participants at the baseline visit (vs $6 \%$ in controls, $P=0.12$ ) and $25 \%$ of tetraplegic participants at the 1 -year visit (vs $5 \%$ of controls $P=0.11$ ). Analyses were not carried out on paraplegics and immobilised controls at 1 year because of the small numbers of participants providing data.

Pulse rates: baseline

Paraplegics had significantly higher $24-\mathrm{h}$, day and night pulse rates as compared with each of the other groups (Table 3). Considering high and low paraplegia separately, 24-h, day and night pulse rates were significantly higher in both high paraplegics $(85.4 \pm 4.6$ b.p.m. over 24 h, $89.1 \pm 5.5$ b.p.m. in day, $77.3 \pm 4.4$ b.p.m. at night) and low paraplegics $(82.3 \pm 2.2$ b.p.m. over $24 \mathrm{~h}, 88.7 \pm 2.0$ b.p.m. in day, $72.9 \pm 2.7$ b.p.m. at night) as compared with each of the other groups, with the exception that night pulse rates did not differ significantly between low paraplegics and immobilised controls. The pulse rate was significantly lower at night than in the day within each group.

Urine output rates: baseline

In control participants, the urine output rate at baseline was significantly lower at night than during the day (Figure 2). However, the rate of urine output did not fall at night compared with the day in the other three groups. Similarly, there was also no significant 

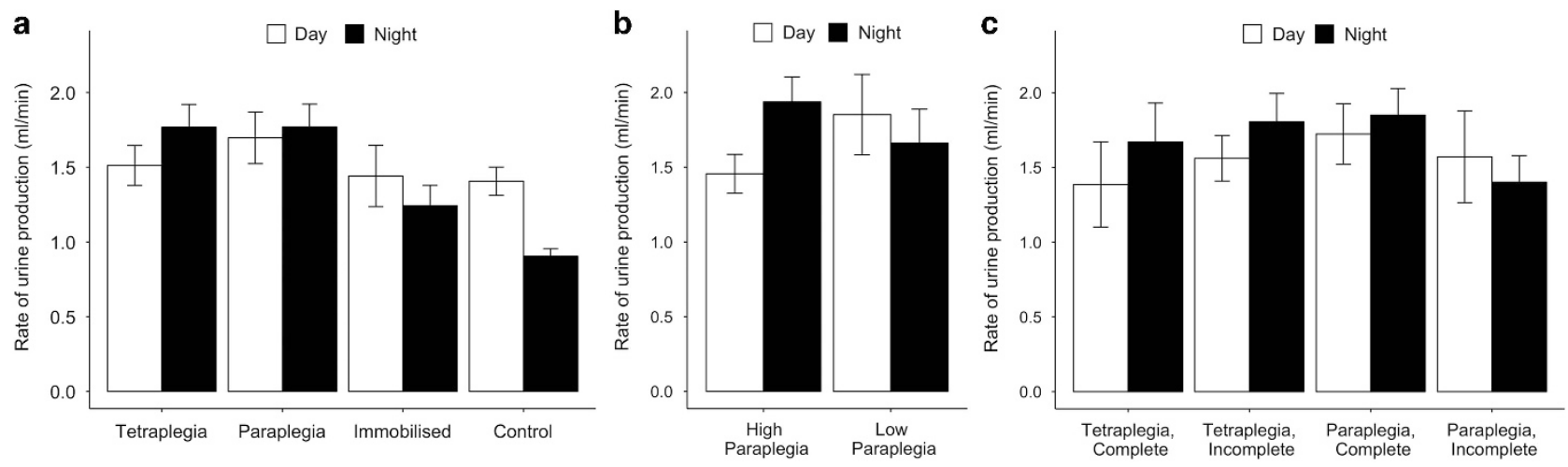

Figure 2 Day and night urine production rates at baseline for (a) tetraplegics $(n=26)$, paraplegics $(n=27)$, immobilised controls ( $n=7)$ and mobilising controls $(n=36), P<0.001$ for day vs night rate of urine production in the control group; (b) high $(n=10)$ and low $(n=17)$ paraplegics; (c) complete $(n=7)$ and incomplete $(n=18)$ tetraplegics and complete $(n=23)$ and incomplete $(n=5)$ paraplegics. Error bars represent s.e.m.

difference between the day and the night rate of urine output in high or low paraplegia and in complete or incomplete SCI.

\section{Correlation between $\mathrm{BP}$ and urine output rates}

The night:day SBP correlated with the night:day urine output rates $\left(r^{2}=0.30, \quad P<0.0001\right)$ when all participants were considered. The correlation was present in the tetraplegics $\left(r^{2}=0.26, P<0.0001\right)$ but not in other groups.

\section{Changes over time}

Nocturnal dipping of BP (expressed as night:day SBP) was compared at baseline and 1 year for each group, using a linear mixed model described above. For the control group, immobilised participants and tetraplegics, the night:day SBP did not differ significantly between visits $(P=0.846, P=0.182$ and $P=0.605$, respectively). For the paraplegic group, there was a significant difference between baseline and 1 year $(94 \pm 1.5 \%$ vs $88 \pm 2.2 \%, P=0.0143)$. There was no significant difference in nocturnal dip in the five assessments over a year in the tetraplegic group. The loss of diurnal variation in urine output rate persisted in both the tetraplegic $(n=6)$ and paraplegic $(n=6)$ participants who completed this measurement at the 1-year assessment.

\section{Symptom scores}

Symptom scores at baseline were significantly different between the four study groups. Median scores were 3, 2, 2 and 0 for tetraplegics, paraplegics, immobilised controls and mobilising controls, respectively ( chi $^{2}=18.26, \mathrm{df}=3, P<0.001$ ). Scores were not significantly different between high and low paraplegia, complete and incomplete tetraplegia or complete and incomplete paraplegia (data not shown).

Symptom scores were completed at baseline and 1 year in 14 tetraplegics, 10 paraplegics, 5 immobilised controls and 26 mobilising controls. Although significant differences were noted across the four groups at baseline $\left(\mathrm{chi}^{2}=8.616, \mathrm{df}=3, P=0.0349\right)$, they were not significantly different at 1 year $\left(\mathrm{chi}^{2}=1.162, \mathrm{df}=3, P=0.762\right)$. Symptom scores did not differ significantly between baseline and 1 year for tetraplegics $(n=14)(P=0.136)$.

At baseline, there was a weak monotonic relationship between dizziness and the nocturnal dipping ratio in the study population as a whole (rho $=0.256, P=0.0069$ ) but not within each group.

\section{DISCUSSION}

This study examined the haemodynamic effects of SCI as a function of time after injury. Previous studies of cardiovascular abnormalities in SCI have predominantly been cross-sectional, conducted in chronic SCI, examined limited numbers of individuals (usually about 10 participants per SCI group) or used restricted entry criteria such as excluding people with 'orthostatic dysregulation' or 'cardiovascular disease' or including only complete SCI. ${ }^{8-11}$ The present study recruited large groups of tetraplegic and paraplegic patients from consecutive admissions to a state-based spinal cord service. This is the first prospective study examining ambulatory BPs soon after SCI and with repeated ABPM measurements over the first year of SCI to assess the persistence of any changes over time.

This study confirms the clinical impression that BP in tetraplegic participants is lower than in mobilising control participants in early SCI, after adjusting for gender. In previous studies, 24-h systolic and diastolic BP have not consistently differed between the tetraplegia and control groups, perhaps because participants with low BP symptoms were excluded, because they were underpowered to detect such a difference or because they were conducted on more established SCI. ${ }^{10,11}$ Another contributing factor may have been the inclusion of female participants in the control group, who usually have lower pressures than male participants. Indeed, when our analysis was not adjusted for gender, we were unable to detect an effect of tetraplegia on SBP.

Diurnal BP variation, particularly in tetraplegics, was profoundly and persistently disrupted for the year following acute SCI. The extent of the disruption meant the majority of tetraplegics had night pressures which exceeded day pressures, and one quarter had nocturnal hypertension. A proportion of paraplegics were similarly affected, especially those with lesions above T6. In paraplegics, there appeared to be improvement over time, so that at 1 year the average night:day SBP ratio was within the dipper category, whereas it was in the non-dipper category at baseline. Our findings in the paraplegic group are in keeping with observations in rat models of thoracic SCI, in which an initial loss was followed by return of diurnal BP variation, compared with pre-SCI patterns. ${ }^{23,24}$ In the general population, including normotensive populations, abnormal diurnal variation (night:day SBP >90\%) and elevated nocturnal BPs have independently been associated with poorer cardiovascular prognoses. ${ }^{25-28}$ The contribution of abnormal diurnal BP to the high rate of cardiovascular disease seen in the SCI population ${ }^{1-4}$ remains to be determined. 
Changes in diurnal BP were paralleled by changes in diurnal urine production, but there was only a weak correlation with orthostatic symptoms. This is consistent with recent report by Hubli et al. ${ }^{13}$ that self-reported symptoms of hypotension did not correlate with episodes of hypotension detected by ABPM. In addition, in keeping with previous studies, we found that both high and low paraplegia, but not tetraplegia, caused the pulse rate to be higher than in control participants. ${ }^{11,29}$ This has been observed in both 24 -h recordings and clinic measurements, but a clear explanation has not yet been determined. ${ }^{11,29,30}$ Vagal function is expected to be normal in SCI, and cardiac autonomic function is expected to be normal in low paraplegia and partially affected in high paraplegia. However, in SCI, there is some suggestion of a shift towards increased sympathetic activity or altered autonomic balance-as evidenced by increased plasma catecholamine levels in low paraplegics ${ }^{11,29,31,32}$ and findings suggestive of vagal pathology in SCI in both human and animal studies. ${ }^{11,33,34}$

Our study included a control group consisting of otherwise healthy individuals who were immobilised in hospital while awaiting orthopaedic surgery-conditions unrelated to spinal cord function. Broadly speaking, immobilised participants had BP patterns that were similar to the mobilising control participants. This suggests that abnormalities of diurnal variation of BP and pulse rate observed in SCI were not a consequence of the experiences the SCI participants shared with immobilised control participants such as immobility, hospitalisation and pain.

Sympathetic fibres leave the spinal cord to innervate effector organs between T1 and L2. ${ }^{35}$ We have made several observations supporting the concept that interruption of sympathetic fibres is more important than immobility as a mechanism contributing to BP abnormalities observed in SCI, including the following: the night:day SBP of the immobilised controls did not differ from mobilising controls; low paraplegia was associated with a lower degree of disruption of diurnal variation compared with tetraplegia; diurnal variation of tetraplegics did not change over time despite the fact that they would have spent more time mobilising as they progressed with rehabilitation; and BP and urine output patterns of tetraplegic patients resembled those seen in other diseases of the autonomic nervous system such as pure autonomic failure, diabetic autonomic neuropathy or disease of the extra-pyramidal system. ${ }^{36-39}$

Nocturnal polyuria in SCI has been attributed to redistribution of fluid arising from the impaired autonomic mechanisms. The loss of vascular tone and muscle tone in the lower limbs results in venous pooling and oedema during the day in the upright position, whereas reversal of this process at night results in increased $\mathrm{BP}$ and reduced antidiuretic hormone levels, leading to diuresis and nocturnal polyuria. ${ }^{40,41}$ Studies in a rat spinal contusion model of SCI have observed polyuria in the range of mild-to-severe impairment in locomotion, which did not improve with increased muscle pump activity by step training, suggesting that other mechanisms contribute to the polyuria. ${ }^{42}$ It has previously been noted in separate studies in humans that there is a loss of diurnal variation in BP in tetraplegics, and a loss of diurnal variation of urine production in both tetraplegia and paraplegia..$^{8-11,40}$ Our study examined these parameters in the same population and observed a weak correlation between the diurnal variation of $\mathrm{BP}$ and that of urine production in tetraplegics but not in paraplegics. This observation is in agreement with previous studies, suggesting that the regulation of urine production in SCI is determined by factors in addition to the result of the motor effects of SCI, posture and BP.

Obstructive sleep apnoea is associated with abnormal diurnal BP variation in the able-bodied population and is therefore another potential cause of abnormal diurnal BP variation in SCI-in whom sleep disordered breathing is an almost ubiquitous finding. ${ }^{43,44}$ However, within the limitation of the small numbers in our study, we were unable to identify an association between abnormalities in diurnal BP and the presence of sleep apnoea in patients with SCI.

Our study included serial observations during a time when participants underwent intensive rehabilitation. Although rehabilitation is associated with a substantial increase in mobility and time spent upright, diurnal BP patterns did not improve in tetraplegic participants. In contrast, the abnormal diurnal variation of paraplegic participants showed improvement at 1 year compared with baseline and was not significantly different from the control group at the 1-year follow-up. This observation might explain why our recent study, ${ }^{14}$ which was performed in paraplegics soon after injury, reported a loss of diurnal variation in paraplegic patients, which was different from most other investigators who had performed their studies in participants with more established injuries. ${ }^{8,10,11}$

Factors such as physical fitness and sleep-disordered breathing are probably different between tetraplegics and paraplegics, ${ }^{10,45,46}$ but our data do not provide insight as to whether either of these factors may have been a contributor.

\section{CONCLUSION}

The results of this study produce several clinical implications. They emphasise the need for ABPM in SCI to determine cardiovascular risk. However, at least for paraplegic patients, measurement might need to be repeated in order to determine that abnormalities in diurnal variation are persistent. Symptoms did not correlate well with abnormalities of BP and should therefore not be relied upon to detect patients in whom ambulatory monitoring should be performed. Finally, this study, which has demonstrated abnormalities in diurnal $\mathrm{BP}$ and urine output in SCI, raises the possibility that abnormal urine output may be associated with BP abnormalities as has been hypothesised in the able-bodied population with autonomic nervous system disease. ${ }^{39}$

\section{DATA ARCHIVING}

There were no data to deposit.

\section{CONFLICT OF INTEREST}

The authors declare no conflict of interest.

\section{ACKNOWLEDGEMENTS}

This research was supported by a grant from the Transport Accident Commission (TAC) (DP174). MG is supported by a scholarship and stipend through the University of Melbourne and Spinal Research Institute. We thank Dr Sue Finch and the Statistical Consulting Centre, University of Melbourne, for their assistance in statistical analysis.

1 Mathias CJ, Low DA, Frankel HL. Autonomic disturbances in spinal cord lesions. In: Mathias CJ, Bannister R (eds). Autonomic Failure: A Textbook of Clinical Disorders of the Autonomic Nervous System, 5th edn. Oxford University Press: Oxford, UK, 2013, pp 797-817.

2 Groah SL, Weitzenkamp D, Sett P, Soni B, Savic G. The relationship between neurological level of injury and symptomatic cardiovascular disease risk in the aging spinal injured. Spinal Cord 2001; 39: 310-317.

3 Myers J, Lee M, Kiratli J. Cardiovascular disease in spinal cord injury: an overview of prevalence, risk, evaluation, and management. Am J Phys Med Rehabil 2007; 86: $142-152$.

4 Garshick E, Kelley A, Cohen SA, Garrison A, Tun CG, Gagnon D et al. A prospective assessment of mortality in chronic spinal cord injury. Spinal Cord 2005; 43: 408-416.

5 Mathias CJ. Orthostatic hypotension and paroxysmal hypertension in humans with high spinal cord injury. Prog Brain Res 2006; 152: 231-243. 
6 Omboni S, Smit AA, van Lieshout JJ, Settels JJ, Langewouters GJ, Wieling W. Mechanisms underlying the impairment in orthostatic tolerance after nocturnal recumbency in patients with autonomic failure. Clin Sci (Lond) 2001; 101: 609-618.

7 Okamoto LE, Gamboa A, Shibao C, Black BK, Diedrich A, Raj SR et al. Nocturnal blood pressure dipping in the hypertension of autonomic failure. Hypertension 2009; 53: 363-369.

8 Nitsche B, Perschak H, Curt A, Dietz V. Loss of circadian blood pressure variation in complete tetraplegia. J Hum Hypertens 1996; 10: 311-317.

9 Krum H, Louis WJ, Brown DJ, Jackman GP, Howes LG. Diurnal blood pressure variation in quadriplegic chronic spinal cord injury patients. Clin Sci 1991; 80: 271-276.

10 Munakata M, Kameyama J, Kanazawa M, Nunokawa T, Moriai N, Yoshinaga K. Circadian blood pressure rhythm in patients with higher and lower spinal cord injury: simultaneous evaluation of autonomic nervous activity and physical activity. $J$ Hypertens 1997; 15: 1745-1749.

11 Rosado-Rivera D, Radulovic M, Handrakis JP, Cirnigliaro CM, Jensen AM, Kirshblum S et al. Comparison of 24- hour cardiovascular and autonomic function in paraplegia, tetraplegia, and control groups: implications for cardiovascular risk. J Spinal Cord Med 2011; 34: 395-403.

12 Hubli M, Krassioukov AV. Ambulatory blood pressure monitoring in spinal cord injury: clinical practicability. J Neurotrauma 2014; 31: 789-797.

13 Hubli M, Gee CM, Krassioukov AV. Refined assessment of blood pressure instability after spinal cord injury. Am J Hypertens 2015; 28: 173-181.

14 Goh MY, Wong ECK, Millard MS, Brown DJ, O'Callaghan CJ. A retrospective review of the ambulatory blood pressure patterns and diurnal urine production in subgroups of spinal cord injured patients. Spinal Cord 2015; 53: 49-53.

15 Kirshblum SC, Burns SP, Biering-Sorensen F, Donovan W, Graves DE, Jha A et al. International standards for neurological classification of spinal cord injury (revised 2011). J Spinal Cord Med 2011 34: 535-546.

16 Claydon VE, Steeves JD, Krassioukov A. Orthostatic hypotension following spinal cord injury: understanding clinical pathophysiology. Spinal Cord 2006; 44: 341-351.

17 DeVivo MJ, Biering-Sørensen F, New P, Chen Y. Standardization of data analysis and reporting of results from the International Spinal Cord Injury Core Data Set. Spinal Cord 2011: 49: 596-599.

18 O'Brien E, Asmar R, Beilin L, Imai Y, Mallion J-M, Mancia G et al. European Society of Hypertension recommendations for conventional, ambulatory and home blood pressure measurement. J Hypertens 2003; 21: 821-848.

19 Fagard R, Brguljan J, Thijs L, Staessen J. Prediction of the actual awake and asleep blood pressures by various methods of $24 \mathrm{~h}$ pressure analysis. J Hypertens 1996; 14: 557-563.

20 Ohkubo T, Imai Y, Tsuji I, Nagai K, Watanabe N, Minami N et al. Relation between nocturnal decline in blood pressure and mortality. The Ohasama Study. Am J Hypertens 1997; 10: 1201-1207.

21 Head GA, McGrath BP, Mihailidou AS, Nelson MR, Schlaich MP, Stowasser M et al. Ambulatory blood pressure monitoring in Australia: 2011 consensus position statement. J Hypertens 2012; 30: 253-266.

22 Freeman R, Wieling W, Axelrod FB, Benditt DG, Benarroch E, Biaggioni I et al. Consensus statement on the definition of orthostatic hypotension, neurally mediated syncope and the postural tachycardia syndrome. Clin Auton Res 2011; 21: 69-72.

23 West CR, Popok D, Crawford MA, Krassioukov AV. Characterizing the temporal development of cardiovascular dysfunction in response to spinal cord injury. J Neurotrauma 2015; 32: 922-930.

24 Mayorov DN, Adams MA, Krassioukov AV. Telemetric blood pressure monitoring in conscious rats before and after compression injury of spinal cord. J Neurotrauma 2001; 18: 727-736.

25 Hoshide S. Associations between nondipping of nocturnal blood pressure decrease and cardiovascular target organ damage in strictly selected community-dwelling normotensives. Am J Hypertens 2003; 16: 434-438.

26 Ohkubo T, Hozawa A, Yamaguchi J, Kikuya M, Ohmori K, Michimata M et al. Prognostic significance of the nocturnal decline in blood pressure in individuals with and without high 24- h blood pressure: the Ohasama study. J Hypertens 2002; 20: 2183-2189.

27 Fagard RH, Thijs L, Staessen JA, Clement DL, De Buyzere ML, De Bacquer DA. Night-day blood pressure ratio and dipping pattern as predictors of death and cardiovascular events in hypertension. J Hum Hypertens 2009; 23: 645-653.

28 Boggia J, Li Y, Thijs L, Hansen TW, Kikuya M, Björklund-Bodegård K et al. Prognostic accuracy of day versus night ambulatory blood pressure: a cohort study. Lancet 2007; 370: 1219-1229

29 Wecht JM, Zhu C, Weir JP, Yen C, Renzi C, Galea M. A prospective report on the prevalence of heart rate and blood pressure abnormalities in veterans with spinal cord injuries. J Spinal Cord Med 2013; 36: 454-462.

30 Ravensbergen HJC, de Groot S, Post MWM, Slootman HJ, van der Woude LHV, Claydon VE. Cardiovascular function after spinal cord injury: prevalence and progression of dysfunction during inpatient rehabilitation and 5 years following discharge. Neurorehabil Neural Repair 2013; 28: 219-229.

31 Schmid A, Huonker M, Barturen JM, Stahl F, Schmidt-Trucksäss A, König D et al. Catecholamines, heart rate, and oxygen uptake during exercise in persons with spinal cord injury. J Appl Physiol 1998; 85: 635-641.

32 Schmid A, Huonker M, Stahl F, Barturen JM, König D, Heim M et al. Free plasma catecholamines in spinal cord injured persons with different injury levels at rest and during exercise. J Auton Nerv Syst 1998; 68: 96-100.

33 Inoue K, Ogata H, Hayano J, Miyake S, Kamada T, Kuno M et al. Assessment of autonomic function in traumatic quadriplegic and paraplegic patients by spectral analysis of heart rate variability. J Auton Nerv Syst 1995; 54: 225-234.

34 Herrity AN, Petruska JC, Stirling DP, Rau KK, Hubscher $\mathrm{CH}$. The effect of spinal cord injury on the neurochemical properties of vagal sensory neurons. Am J Physiol Regul Integr Comp Physiol 2015; 308: R1021-R1033.

35 Nervous regulation of the circulation, and rapid control of arterial pressure. In: Hall JE (ed). Guyton and Hall Textbook of Medical Physiology. 12th edn. Saunders: Philadelphia, PA, USA, 2011, pp 201-211.

36 Senard JM, Chamontin B, Rascol A, Montastruc JL. Ambulatory blood pressure in patients with Parkinson's disease without and with orthostatic hypotension. Clin Auton Res 1992; 2: 99-104.

37 Mathias CJ, Fosbraey P, da Costa DF, Thornley A, Bannister R. The effect of desmopressin on nocturnal polyuria, overnight weight loss, and morning postural hypotension in patients with autonomic failure. Br Med J (Clin Res Ed) 1986; 293 : 353-354.

38 Schmidt C, Berg D, Prieur S, Junghanns S, Schweitzer K, Globas C et al. Loss of nocturnal blood pressure fall in various extrapyramidal syndromes. Mov Disord 2009; 24: 2136-2142.

39 Shibao C, Gamboa A, Abraham R, Raj SR, Diedrich A, Black B et al. Clonidine for the treatment of supine hypertension and pressure natriuresis in autonomic failure. Hypertension 2006; 47: 522-526.

40 Kilinç S, Akman MN, Levendoglu F, Ozker R. Diurnal variation of antidiuretic hormone and urinary output in spinal cord injury. Spinal Cord 1999; 37: 332-335.

41 Szollar S, Dunn K, Brandt S, Fincher J. Nocturnal polyuria and antidiuretic hormone levels in spinal cord injury. Arch Phys Med Rehabil 1997; 78: 455-458.

42 Ward PJ, Hubscher $\mathrm{CH}$. Persistent polyuria in a rat spinal contusion model. J Neurotrauma 2012; 29: 2490-2498.

43 Berlowitz DJ, Brown DJ, Campbell DA, Pierce RJ. A longitudinal evaluation of sleep and breathing in the first year after cervical spinal cord injury. Arch Phys Med Rehabil 2005; 86: 1193-1199.

44 Berlowitz J, Spong J, Gordon I, Howard E, Brown J. Relationships between objective sleep indices and symptoms in a community sample of people with tetraplegia. Arch Phys Med Rehabil 2012; 93: 1246-1253.

45 Sankari A, Bascom A, Oomman S, Badr MS. Sleep disordered breathing in chronic spinal cord injury. J Clin Sleep Med 2014; 10: 65-72.

46 Burns SP, Kapur V, Yin KS, Buhrer R. Factors associated with sleep apnea in men with spinal cord injury: a population-based case-control study. Spinal Cord 2001; 39: $15-22$ 